\title{
Éléments pour une théorie de l'ESS : les cas du Brésil et de la France
}

\section{Towards a social and solidarity economy theory: The Brazilian and French experiences \\ Elementos para una teoría de la ESS: los casos del Brasil y de Francia}

\section{Magali Zimmer et Carolina Orquiza Cherfem}

Numéro 336, avril 2015

URI : https://id.erudit.org/iderudit/1030159ar

DOI : https://doi.org/10.7202/1030159ar

Aller au sommaire du numéro

Éditeur(s)

Association Recma

ISSN

1626-1682 (imprimé)

2261-2599 (numérique)

Découvrir la revue

Citer cet article

Zimmer, M. \& Cherfem, C. O. (2015). Éléments pour une théorie de l’ESS : les cas du Brésil et de la France. Revue internationale de l'économie sociale, (336),

36-49. https://doi.org/10.7202/1030159ar
Résumé de l'article

Cet article analyse les différentes conceptualisations de l'économie sociale et solidaire (ESS) issues des milieux académiques brésilien et français, et plus particulièrement la manière dont elles appréhendent la place de l'ESS dans la société et le changement social. L'objectif est d'identifier les divergences et les similitudes à travers une comparaison des débats théoriques dans les deux pays. Du côté brésilien, les principaux auteurs mettent en avant l'autogestion comme principe entrant en contradiction avec le reste de la société. Cependant, le rôle de l'Etat apparaît important pour le développement de ce secteur. Du côté français, c'est à la fois l'autonomie et le rôle de l'Etat qui sont valorisés. La synthèse met en évidence la contribution des conceptualisations propres à chaque pays dans l'élaboration d'un nouveau modèle culturel au sens où l'entend Alain Touraine. 


\title{
ELÉMENTS POUR UNE THÉORIE DE L'ESS : LES CAS DU BRÉSIL ET DE LA FRANCE
}

par Magali Zimmer* et Carolina Orquiza Cherfem**

\author{
* Doctorante au Conservatoire \\ national des arts et métiers \\ (Cnam) à Paris. Mél. : magali_ \\ zimmer@yahoo.fr. \\ ** Doctorante à l'université \\ de Campinas (Unicamp) au \\ Brésil. Mél. : carolinacherfem \\ @yahoo.com.br.
}

\begin{abstract}
Cet article analyse les différentes conceptualisations de l'économie sociale et solidaire (ESS) issues des milieux académiques brésilien et français, et plus particulièrement la manière dont elles appréhendent la place de l'ESS dans la société et le changement social. L'objectifest d'identifier les divergences et les similitudes à travers une comparaison des débats théoriques dans les deux pays. Du côté brésilien, les principaux auteurs mettent en avant l'autogestion comme principe entrant en contradiction avec le reste de la société. Cependant, le rôle de l'Etat apparaît important pour le développement de ce secteur. Du côtéfrançais, c'est à la fois l'autonomie et le rôle de l'Etat qui sont valorisés. La synthèse met en évidence la contribution des conceptualisations propres à chaque pays dans l'élaboration d'un nouveau modèle culturel au sens où l'entend Alain Touraine.
\end{abstract}

Towards a social and solidarity economy theory: The Brazilian and French experiences This article examines the different conceptions of the social and solidarity economy (SSE) in Brazilian and French academic circles and, more particularly, how they view the SSE's role in society and social change. The aim is to identify the differences and similarities by comparing the theoretical literature in the two countries. On the Brazilian side, the main authors stress self-management as a principle in contradiction with the rest of society. However, government seems to play an important role in the growth of this sector. On the French side, independence is emphasised, but government's role is also stressed. The comparison highlights the contribution of each country's conception to the development of a new cultural model in Touraine's sense.

\section{Elementos para una teoría de la ESS : los casos del Brasil y de Francia}

Este articulo analiza las diversas conceptualizaciones de la ESS producidas en los medios académicos, brasileño y francés, y más particularmente como ellas captan el lugar de la ESS en la sociedad y el cambio social. El objetivo es identificar divergencias y analogías a través de una comparación de los debates teóricos entre los dos países. Por parte de Brasil, los principales autores hacen hincapié en la autogestión como principio entrando en contradicción con el resto de la sociedad. Sin embargo, el papel del Estado aparece importante para el desarrollo de este sector. Por parte de Francia, es la autonomía que es valorizada, insistiendo también sobre el papel del Estado. La síntesis pone de relieve el aporte de las conceptualizaciones propias a cada país en la elaboración de un nuevo modelo cultural en el sentido de Alain Touraine. 
Si l'économie sociale et solidaire (ESS) trouve un nouvel élan dans le débat public en période de crise économique, il convient d'interroger le socle théorique sur lequel elle s'appuie.

Depuis plusieurs décennies, la recherche en ESS s'enrichit des apports de diverses disciplines académiques. En 2013, un ouvrage collectif dressait une synthèse des approches théoriques portant sur l'économie sociale et solidaire au niveau international (Hiez, Lavillunière, 2013). Dense, il mêlait des contributions théoriques de l'Angleterre, de la Belgique, du Brésil, de la France, du Québec et de la Suisse. Notre approche se limitera à la comparaison de la France et du Brésil, afin, d'une part, de clarifier le contenu des théories dans ces deux pays et, d'autre part, de comprendre la place attribuée à l'ESS à l'intérieur de la société et son rôle dans le changement social. Des dénominations encore nombreuses parsèment ces deux traditions théoriques. Au Brésil, l'économie populaire est présentée comme étant plus ancienne et relativement dénuée de perspective politique, comparée à l'économie solidaire, qui s'inscrit dans son prolongement d'un point de vue chronologique mais est associée à des questions de reconnaissance des droits sociaux, notamment dans le domaine du traitement des déchets urbains et dans celui de l'éducation (Carvalho de França Filho, 2003). Au fur et à mesure que les travaux académiques se sont développés, l'expression « économie solidaire » s'est diffusée dans le débat public au Brésil, avec l'essor des incubateurs universitaires.

A l'opposition entre économie populaire et économie solidaire au Brésil se superpose l'opposition entre économie sociale et économie solidaire en France. Sans s'attacher à comparer systématiquement les pratiques socioéconomiques et les dénominations associées, il s'agira de saisir la diversité des approches théoriques qui existent dans les deux pays, en suivant comme fil conducteur la place accordée à l'ESS ${ }^{(1)}$ dans la société par

(1) Quand nous nous référerons à un auteur en particulier, nous reprendrons la dénomination propre à cet auteur («économie solidaire» pourSinger, «socio-économie solidaire » pour Arruda, etc.), mais, lorsque nous élargirons le propos, nous adopterons l'expression «économiesociale et solidaire " qui, bien que propre au contexte français et recouvrant des traditions distinctes, nous permettra d'englober l'ensemble des entités définies par la loi-cadre sur l'ESS du 31 juillet 2014. les différents auteurs. C'est à la restitution de la complexité de ces approches théoriques que s'attelle le présent article, afin de mieux comprendre quels sont les éléments essentiels permettant d'esquisser une théorie commune de l'ESS. Enfin, les différentes conceptions de la contribution de cette dernière au changement social seront analysées dans les deux pays.

Cet article dresse un état des lieux des conceptualisations de l'ESS dans les milieux académiques brésilien et français. L’objet est d'identifier et de clarifier les divergences contenues dans les recherches à travers une comparaison des débats théoriques, en espérant que ceux-ci puissent éclairer les praticiens sur les enjeux actuels du développement de l'ESS dans les deux pays. La délimitation du corpus de textes repose sur deux critères: les auteurs contemporains retenus nous semblaient se distinguer par un grand nombre de publications à caractère théorique et ont contribué de manière substantielle à la construction d'une théorie de l'ESS. Parmi eux, nous avons sélectionné ceux qui nous paraissaient présenter des problématiques comparables, selon deux axes: une attention portée à l'échelle macrosociale du changement social et une réflexion théorique spécifique quant au positionnement de l'ESS dans la société. L'ambition 
de notre article n'est pas de dresser un état des lieux des différentes dénominations existantes et de leur contexte d'apparition, mais plutôt de comprendre comment les auteurs dont les travaux portent sur l'ESS participent, au-delà de l'apparente dispersion, à la construction d'une théorie générale du changement social.

Nous présenterons donc, dans une première partie, le cas du Brésil, puis, dans une seconde partie, celui de la France, et, dans une troisième partie, une synthèse des deux.

\section{Les éléments théoriques brésiliens apportés au débat}

\section{L'économie solidaire, aiguillon de la démocratisation de la société}

L'économiste Paul Singer est un précurseur de l'économie solidaire (ES) au Brésil. Il élabore sa théorie de l'ES en tenant compte des processus historiques à l'œuvre dans les coopératives ouvrières issues des luttes qui émergent au moment de la révolution industrielle, telles que les expériences britanniques du début du XIX ${ }^{\mathrm{e}}$ siècle inspirées par Robert Owen et ses projets de villages coopératifs (Singer, 2000 $a$ ). Le coopérativisme trouve ses origines dans l'expérience de Rochdale, une société coopérative devenue la mère des coopératives modernes en définissant des principes qui font encore référence. Au Brésil, c’est durant les décennies 80 et 90 que le coopérativisme gagne en visibilité, dans un contexte caractérisé par un chômage endémique et une déréglementation de l'économie (Singer, 2003, p. 117). L'ES est alors conçue comme une issue réaliste pour la population exclue, mais est également présentée sous un angle politique de par les valeurs de démocratie, de solidarité, de coopération et d'autogestion qu'elle promeut. Pour Singer, l'autogestion est indissociable de l'ES. Elle fait partie intégrante de l'histoire ouvrière, puisqu'il s'agit de construire un mode de production socialiste reposant sur une organisation collective du travail (Singer, 2003 et 2005). L'auteur s'appuie notamment sur l'expérience yougoslave de 1950, d'où vient l'expression "socialisme autogestionnaire ", ainsi que sur les luttes des travailleurs des années 60 et 70 à l'origine de la révolution des Eillets, en 1974, au Portugal.

Pour Singer, les luttes des travailleurs prennent des formes différentes selon les périodes, et la période actuelle est caractérisée par une coexistence de l'ES et du capitalisme (Singer, 2003), qui rend possible la quête de justice par les acteurs de l'ES, tout en les maintenant immergés dans une société capitaliste. L'auteur pointe dans ses recherches la puissance de la "désaliénation que la propre pratique d'autogestion donne à ses travailleurs ", ainsi que la puissance d'une "éducation critique et désaliénante contre la dégénérescence » qui peut se produire dans l'ES (Singer, 2000b, p. 42). Si l'ES n'apporte pas en soi un nouveau modèle autogestionnaire de société, elle constitue néanmoins les prémices d'une économie socialiste et démocratique. Le modèle de l'ES n'est pas le même que celui d'une économie socialiste planifiée, mais ses pratiques, couplées avec d'autres facteurs, peuvent permettre la construction de cette économie démocratique (Singer, 2000b). Pour l'auteur, l'ES pourrait être l'embryon de l'économie socialiste autogérée. 
Le sociologue Luiz Inácio Gaiger $(2000,2004)$ quant à lui ne conçoit pas l'ES comme un nouveau mode de production. Se référant à Marx, il définit le concept de mode de production comme un état de développement matériel et humain rendu possible dans un certain contexte historique et qui émerge " en cohérence avec un état historique du développement des forces productives " (Gaiger, 2004, p. 6). A l'intérieur d'un mode de production se trouvent plusieurs «formes sociales de production " (forme familiale, de voisinage...), qui peuvent coexister dans le même système, et la difficulté de cette coexistence est le principal défi posé à ces formes d'organisation, en particulier du point de vue technologique, éducatif et politique. Non pas forme hégémonique, mais forme complémentaire des autres formes sociales de production, telle semble être la place accordée par l'auteur à l'ES dans la société. De cette approche, il résulte aussi que l'utilité sociale de l'ES doit être examinée en partant du point de vue des besoins des personnes réunies dans les projets, et non pas seulement à partir de l'observation de l'accumulation du capital (Gaiger, 2000, p. 181). Concernant le rapport à l'Etat, Gaiger soutient que toutes les organisations perçoivent des aides étatiques et qu'aucun secteur économique ne peut être indépendant financièrement, pas même l'ES (Gaiger, 2000, p. 180).

L'économiste Marcos Arruda est en accord avec l'idée d'émancipation dans l'ES, mais estime qu'elle doit être radicalisée pour surmonter le capitalisme. Le sens étymologique du terme «économie » désigne la "gestion de la maison ", c'est-à-dire la gestion de notre corps, de l'espace dans lequel nous vivons avec notre famille, de notre communauté, de notre ville, de notre pays ou planète (Arruda, 2005). Dans cette perspective, l'économie repose sur le principe de suffisance. L'auteur constate cependant que le terme "économie » a perdu son sens premier dans le contexte de l'économie capitaliste et suggère donc l'utilisation du terme "socio-économie solidaire ", pour mettre l'accent sur la dimension sociale (à laquelle renvoie le préfixe " socio-») tout autant que sur la dimension solidaire. Cette conceptualisation représente une nouvelle génération de pratiques en ES au Brésil, marquée par des revendications non seulement économiques, mais aussi féministes et environnementales. Pour la définir, Arruda (2003) distingue quatre aspects, qu'il pose comme fondamentaux et qui constituent l'idéal-type de la socioéconomie solidaire, à savoir: la préservation de l'environnement, la prise en compte de l'être humain comme un être solidaire par nature, le féminin comme dimension qui doit être intégrée dans l'économie et l'éducation en tant que praxis essentielle pour la construction de l'être humain. Les relations entre ces quatre éléments doivent conférer un pouvoir d'émancipation à la socio-économie solidaire.

Dans le modèle économique dominant, celle-ci n'occupe que fort peu de place (Arruda, 2003), si bien que les pratiques de l'ES actuelles ne peuvent pas parvenir «à offrir une alternative à l'économie systémique du capital» (2003, p. 240). L'une des raisons invoquées par l'auteur tient à la taille de ces projets, qui sont d'ordre micro-économique et se révèlent incapables de surmonter le système capitaliste. La coexistence doit rester provisoire jusqu'à l'abolition du capitalisme. En analysant les politiques de l'ES du Secrétariat national à l'économie solidaire au Brésil, l'auteur conclut que le gouvernement 
ne prépare pas un projet viable susceptible d'atteindre ce but. Compte tenu de cela, il suggère que les acteurs politiques qui participent aux Forums sociaux mondiaux commencent à s'organiser pour exercer une pression sur l'Etat (Arruda, 2000, p. 222).

\section{Vers une prudente coexistence des principes économiques}

D’autres auteurs brésiliens comprennent l'ES comme un ensemble d'initiatives importantes à étudier d'un point de vue à la fois intellectuel et sociétal, mais dans une perspective critique. Ils mettent l'accent sur son potentiel de résorption du chômage et du travail informel, et non sur la possibilité de construire une société démocratique.

Dans une recherche récente développée dans l'Etat de São Paulo, une équipe de sociologues composée de Leite, Lima et Araújo (2011) a analysé les différents aspects de l'ES en vue d'explorer la diversité de ses formes d'organisation. A travers des entretiens menés dans des coopératives de petite taille issues d'usines récupérées (comprenant les secteurs de la fabrication, de l'artisanat et de l'alimentation) et dans des coopératives de recyclage, les auteurs ont relevé une très grande diversité de formes de l'ES, certains aspects étant parfois même contradictoires. Ils mettent en évidence l'écart important qui sépare la théorie défendue par les dirigeants du mouvement de l'ES du vécu des travailleurs appartenant à ces organisations. Ils reconnaissent néanmoins les possibilités offertes par ces formes d'organisation économique pour la constitution d'un mouvement social, en présentant des cas de « résistance des travailleurs » non négligeables. Cette résistance se caractérise par le refus de perdre son emploi, avec la récupération d'usines, par la volonté de lutter contre le chômage, par le développement de la capacité d'agir des travailleurs ou encore par la recherche de "formes d'intégration sociale fondées sur la solidarité entre les travailleurs » (Leite, 2011, p. 2-6).

Les sujets étudiés par le sociologue Faria (2009) s’inscrivent dans la même orientation, avec une attention supplémentaire portée à la nécessité de repenser le concept d'ES en partant des expériences développées sur le terrain et en prenant soin de ne pas généraliser trop hâtivement. L'auteur souligne que la plupart des travailleurs de cette économie ne s'unissent pas pour créer une alternative au capitalisme, mais pour remédier aux conditions et aux conséquences pratiques de l'inégalité du capital, telles que le chômage (Faria, 2009). Il met l'accent sur l'erreur conceptuelle de ce qu'il appelle l'« autogestion sociale ». Cette erreur consiste à inclure l'ES dans le champ de l'autogestion sociale, comme le fait Singer, alors qu'elle s'inscrit pour Faria dans un champ qu'il nomme l'« autogestion partielle ou collectiviste ». Ces expériences de l'autogestion partielle sont appelées "organisations sociales productives (OSP) » et seraient une source de confrontation avec le mode de production capitaliste, et non un système de remplacement, en d'autres termes elles existent par et pour cette opposition. Après les expériences des soviets en Russie, de la révolution espagnole ou de la révolution des Eillets au Portugal en 1974, qui se conclurent par un échec, plus personne n'entendit parler de l'autogestion. C'est en 1990 avec l'économie solidaire que le terme "autogestion " est réapparu au Brésil pour désigner les organisations des travailleurs autonomes au sein du capitalisme, 
apportant une nouvelle façon de gérerdes projets sans pour autant s'inscrire dans une visée de transformation complète de la société. C'est en prenant en compte les perspectives de ces diverses expériences que l'auteur a préféré parler des OSP en termes d'autogestion partielle. Il définit au moins trois critères permettant de caractériser la notion d'autogestion partielle: surmonter la division du travail, qui distingue les décideurs et les exécutants; permettre l'autonomie de décision de chaque unité d'activité; favoriser la participation des travailleurs dans toutes les sphères de l'organisation. Les OSP se construisent ainsi autour de l'objectif de modifier les rapports de pouvoir entre les travailleurs à l'intérieur d'une organisation productive, ce qui induit une contradiction entre ces formes d'organisation et le système capitaliste. Cependant, Faria (2009) constate qu'elles sont pourvues d'une logique spécifique et qu'elles n'ont pas l'intention de se substituer au capitalisme, car elles en constituent justement sa contradiction. La principale question n'est donc pas de savoir si les OSP peuvent ou non transformer la société capitaliste, mais quelles sont les avancées et les particularités qu'elles rendent visibles par leurs contradictions.

\section{Les éléments théoriques français apportés au débat}

\section{L'économie sociale et solidaire, premier pas vers une société plus égalitaire}

Jean-François Draperi, géographe et sociologue de formation, a écrit de nombreux livres retraçant l'histoire de la pensée de l'économie sociale. Nous nous appuyons ici sur l'un de ses derniers ouvrages théoriques majeurs, Comprendre l'économie sociale, pour saisir les fondements de son approche. L'économie sociale est définie comme l'ensemble des «entreprises coopératives, mutualistes et associatives » qui ont "pour finalitéde répondre aux besoins et aux attentes collectives» (Draperi, 2007, p. 7). Les valeurs qui unissent les acteurs de l'économie sociale sont au nombre de quatre: « responsabilité (ou volontariat), égalité, solidaritéet autonomie» (ibid., p. 17). La double qualité constitue, pour Draperi, un principe central d'organisation de toute entreprise d'économie sociale. Il est repris du théoricien du mouvement coopératif, Georges Fauquet, et signifie que les bénéficiaires de l'action sont en même temps les sociétaires de l'entreprise. Il permet d'établir un rapport démocratique entre associés. La "double qualitécomme principe central de l'économie sociale doit être comprise comme un moyen de la finalité éducative et émancipatrice de l'entreprise d'économie sociale » (ibid., p. 20). C'est un principe idéal typique. Il n'est pas à l'œuvre dans de nombreuses associations, telles que celles qui offrent un service à un public spécifique, qui ne peut pas participer au fonctionnement démocratique de l'association. De même, la double qualité n'est pas toujours mise en pratique dans les entreprises coopératives, comme l'auteur le reconnaît lui-même. Les termes "entreprise " ou "entrepreneur " qui sont fréquemment utilisés par l'auteur pour désigner les organisations de l'économie sociale ne dénotent pas une absence de dimension politique. L'entrepreneur en économie sociale est en effet conçu comme « militant » qui «milite pour sa cause, qui est son entreprise et son projet »(Draperi, 2007, p. 39). L'expression 
«entrepreneur militant» est également un idéal-type et constitue à ce titre " un outil d'analyse permettant de dégager la logique démocratique de l'entreprise d'économie sociale » (ibid., p. 40).

Pour Draperi, la différence fondamentale entre la pensée marxiste et la pensée coopérative ne tient pas au caractère utopique, mais plutôt au moyen d'accéder à cette utopie. La pensée coopérative conçoit le changement social non pas en tant qu'événement brusque et violent comme dans la pensée marxiste, mais suivant une démarche pacifiste et gradualiste, car l'économie sociale pense "simultanément le but et les moyens de parvenir à ce but »(Draperi, 2007, p. 76). Loin d'être figée, la pensée de l'économie sociale se constitue autour d'utopies qui évoluent au fil du temps. Au XIX ${ }^{\mathrm{e}}$ siècle, ce sont les phalanstères et les microrépubliques communautaires qui sont le fondement de cette utopie. Puis vient la république coopérative avec Charles Gide et, enfin, dans la seconde partie du $\mathrm{XX}^{\mathrm{e}}$ siècle, le développement local. Le modèle actuel serait ainsi celui de la «méso-république du développement local» (Draperi, 2005, p. 102). La pensée de l'économie sociale actuelle est donc essentiellement marquée par une utopie portée vers le développement local endogène, mais des sédiments des utopies passées restent toujours perceptibles aujourd'hui.

Un autre auteur, Jacques Prades, économiste, a également insisté sur la valeur d'autonomie comme élément central de l'économie sociale. Il met l'accent sur les formes d'autodétermination caractérisées par « la capacité des hommes à prendre en charge collectivement leur histoire» (Prades, 2006, p. 20). Le changement social provient non pas d'une action éducative, mais précisément de cette capacité d'autodétermination. A la différence du précédent auteur, pour Prades les révolutions n'ont pas débuté par l'éducation populaire, mais par une autodétermination spontanée (telle que la Commune de Paris, la révolution russe de 1905 ou le mouvement de 1968 en France et celui de l'autogestion; ibid.). L'économie sociale s'inscrit dans un «combat pourl'autodétermination », ce qui l'oppose à la vision fonctionnelle de Castel notamment.

Dans cette approche reposant sur l'autodétermination, la question de la propriété collective est centrale. Or, d'après Prades, ce débat sur la propriété collective a longtemps été occulté, et il doit être remis au centre des études portant sur l'économie sociale. C'est en effet sous cet angle que l'on peut comprendre la nouvelle économie sociale. La "propriété collective " a pris la forme en Europe de l'Est d'une propriété étatique et, en Europe de l'Ouest, d'une concentration du pouvoir financier. L'hypothèse de l'auteur est qu'entre les deux existe un type de propriété collective des biens d'usage et des biens de production qui permet aux individus de se prendre en charge. Cette position tend à privilégier, à l'intérieur de l'économie sociale, l'étude de "pratiques d'agglomération » qui durent et ne relèvent pas d'une échelle expérimentale (telle que les circuits courts ou les entreprises alternatives). A partir des années 70, parallèlement à la banalisation des coopératives et à l'institutionnalisation des mutuelles, un regain du mouvement associatif se produit et sera dénommé "économie solidaire ". Prades estime cependant que ce regain associatif, qui se déploie à petite échelle et dont le développement repose sur le don, restera marginal dans la société salariale (Prades, 2006). Si le plus grand danger qui menace notre société tient 
à la perte du sens de l'utopie, il convient alors de le retrouver à travers une conceptualisation de l'économie sociale ayant une plus forte dimension politique (Prades, 2013).

Face à la crise de l'Etat-providence, de nombreuses associations ont tenté d'apporter une réponse au problème du chômage de masse, contre lequel l'Etat s'est révélé incapable de lutter. Leurs interventions ont pris diverses formes: les centres d'aide par le travail, les ateliers protégés, les sociétés coopératives de production, les entreprises d'insertion, les association intermédiaires, les régies de quartier, etc. Ces initiatives se développent essentiellement dans le secteur des services de proximité et émergent en réponse à des demandes sociales liées à la qualité de la vie, à la socialisation des enfants en bas âge, à l'intégration des personnes âgées, etc. (Eme, Laville, 1988; Laville, 2005). Le terme « économie solidaire » est alors proposé par Laville et Eme pour les désigner. Pour conceptualiser l'émergence d'une initiative solidaire, la réciprocité est considérée comme essentielle, toute initiative solidaire partant d'une «impulsion réciprocitaire» (Laville, 1994, p. 74). Néanmoins, elles ne peuvent s'inscrire dans la durée que si elles réussissent à combiner "ressources marchandes obtenues par le produit des ventes, ressources non marchandes émanant de la redistribution, ressources non monétaires issues de contributions volontaires »(Laville, 2001). L’hybridation des ressources est donc considérée comme une condition nécessaire permettant aux initiatives solidaires de perdurer. Cette notion d'hybridation des ressources s'appuie sur les travaux de Polanyi. Le concept d'économie plurielle proposé par Laville provient de la combinaison des trois grands types d'économie distingués par Polanyi: le marché (l'économie marchande), la redistribution (l'économie non marchande) et la réciprocité (l'économie non monétaire). Le caractère pluriel de l'économie s'oppose à une vision de l'économie réduite au seul principe marchand. Une part importante de la vie économique, notamment celle qui est régie par la solidarité, peut alors être prise en compte. Dans cette conceptualisation de l'économie solidaire, l'Etat joue un rôle central dans le développement de ces initiatives, mais ces dernières exercent aussi une influence sur l'Etat à travers leur capacité d'interpellation.

\section{L'économie sociale et solidaire, supplétif de l'Etat social}

A l'opposé des approches précédentes, qui considéraient de manière explicite ou implicite les entités appartenant à l'économie sociale et solidaire comme des vecteurs d'émancipation, d'autres auteurs portent un regard plus critique sur leurs développements. Ainsi, le sociologue Matthieu Hély a étudié l'évolution de l'emploi dans le secteur associatif au cours des trente dernières années. Selon lui, le nombre de salariés employés par une association a fortement augmenté, de 600000 au début des années 80 à un million et demi aujourd'hui (Hély, 2009, p. 2). Il ne cesse de grandir et pourrait à terme dépasser le nombre de fonctionnaires. L'emploi dans le secteur associatif crôit en effet à une vitesse deux fois plus rapide que celle de l'emploi dans la fonction publique. Pour Hély, "l'importance prise par le monde associatif résulte avant tout d'une politique de brouillage des frontières entre privé et public et d'une déstabilisation profonde des relations salariales »(Hély, 2009, p. 4). Selon lui, 
le secteur associatif est perçu comme étant dépourvu de dynamique propre qui permette l'élaboration d'un projet collectif. Appréhendé avant tout comme un marché du travail, il s'inscrit dans un contexte de refonte de l'Etat social. L'événement marquant de ces trente dernières années est en effet pour Hély la décentralisation de l'action étatique. Celle-ci a débuté dès 1975 avec l'encouragement de l'établissement par l'Etat de conventions avec les associations habilitées à recevoir des bénéficiaires de l'action sociale. Les effets de cette décentralisation sur le secteur associatif sont étudiés à partir de données statistiques sur l'emploi salarié, en tenant compte du fait que les catégories des institutions qui produisent des données statistiques sont inadaptées au monde associatif. La véritable tendance qui résulte de cette décentralisation réside dans le processus de conversion des associations en " entreprises associatives » (Hély, 2009, p. 21). A côté de la banalisation ayant marqué le développement des coopératives, de l'institutionnalisation ayant caractérisé les mutuelles dans les années 80 , ce sont désormais les associations qui se sont à leur tour éloignées des principes fondateurs, sous l'effet d'une mise en concurrence avec le secteur marchand.

Le concept d'" entreprise associative » fait référence au passage de l'association composée de bénévoles à l'association employeur d'au moins un salarié. Parmi les entreprises associatives, quatre types peuvent être dégagés de l'analyse statistique: l'entreprise associative gestionnaire, l'entreprise associative partenaire, l'entreprise associative marchande et l'entreprise associative mécénale (Hély, 2009). Refusant d'employer l'expression «économie solidaire », cet auteur conçoit le monde associatif comme un ensemble d'entités économiques participant pleinement au marché, avec une capacité d'interpellation dans l'espace public très faible. Pour Hély, le développement du secteur associatif participe à l'affaiblissement de l'Etat social, car l'emploi dans le secteur croît au détriment de l'emploi public et contribue à la généralisation de l'emploi atypique.

\section{Synthèse}

Les conceptions du changement social et le rôle potentiel de transformation attribué à l'économie sociale et solidaire sont variés et nourrissent un débat en France et au Brésil, dont nous tenterons de souligner les enjeux dans cette partie. Ce débat est lié à la place ou fonction assignée à l'ESS dans la société et recouvre des divergences épistémologiques fortes entre, d'un côté, des perspectives émancipatrices et, de l'autre, des perspectives critiques ou pragmatiques. Un éclaircissement des termes de la discussion permet de clarifier les formes archétypales de projets de transformation de la société en jeu. Au Brésil, par exemple, ce débat oppose les théoriciens qui conçoivent l'économie solidaire sous un angle émancipateur avec une attention portée aux formes d'autogestion (Singer, 2000 $a, 2000 b$ et 2003; Gaiger, 2000 et 2004; Arruda, 2003 et 2005) à ceux qui, au contraire, mettent l'accent sur sa faculté à participer à la résorption du chômage (Faria, 2009; Leite et al., 2011). En France, ce débat dresse les auteurs mettant au centre de leurs études la dimension éducative (Draperi, 2007), l'autodétermination (Prades, 2006) ou la réciprocité (Laville, 2007) contre ceux qui suivent 
une perspective critique proposant d'interpréter le délitement de l'Etat social comme principal facteur explicatif de l'augmentation d'emplois de moindre qualité dans le secteur de l'ESS (Hély, 2009).

Pour affiner la comparaison entre les conceptualisations françaises et brésiliennes, nous reviendrons, dans un premier temps, sur le rôle attribué à l'Etat et soulèverons, dans un second temps, les implications culturelles des changements sociaux auxquels l'ESS prend part, au niveau tant organisationnel qu'individuel.

Le rapport à l'Etat des entités appartenant à l'ESS est l'objet d'un questionnement théorique ancien. Comment les auteurs mentionnés dans cet article abordent-ils ce débat? Pour un grand nombre d'entre eux, l'Etat a un rôle essentiel à jouer au Brésil, mais avec des degrés de soutien variables suivant les auteurs. Certains considèrent en effet que l'ES entretient un rapport de complémentarité avec l'Etat, voire de dépendance. A cet égard, nous constatons l'accord existant entre Gaiger et Arruda, tous deux incluant la nécessité d'un soutien de l'Etat à travers l'élaboration de politiques efficaces pour accroître le développement de l'ES. La nécessité d'un soutien de l'Etat est en effet également défendue par Arruda. Un parallèle peut être mené entre Gaiger et Laville au sujet de la conceptualisation du fonctionnement. Gaiger souligne la diversité de «formes sociales de production » présentes dans une même entité, celles-ci reposant sur la solidarité, le capitalisme ou la famille. Cette pluralité des formes sociales de production fait écho à la pluralité des principes économiques que sont la réciprocité, le marché et la redistribution mis en avant par Laville. Dans les deux cas, c'est l'évolution des relations entre ces différentes formes sociales de production ou ces différents principes économiques qui explique le fonctionnement des initiatives solidaires. De cette combinaison découle une relation d'interdépendance étroite vis-à-vis des financements publics, qui est aussi un gage d'équilibre sur le long terme. Non pas recherche de complémentarité, mais surtout recherche d'autonomie, telle est la principale orientation de l'ESS pour toute une autre frange de théoriciens. Selon cette approche, l'ESS s'inscrit dans un mode davantage fédératif que centralisateur du changement social, dans le sens où la liberté et l'autonomie deviennent des éléments essentiels du développement des unités de production. Pour Prades, le débat sur la propriété collective doit être remis au centre des études portant sur l'ESS en vue de permettre aux individus de se prendre en charge indépendamment de l'Etat. Il assigne, de plus, aux études portant sur l'ESS l'objectif de se centrer sur les pratiques d'agglomération (Prades, 2006), seules capables d'apporter la perspective d'un changement social de grande ampleur. De même, pour Draperi, l'économie sociale doit revendiquer "l'indépendance économique de ses entreprises» (Draperi, 2007, p. 28). Au concept d'autonomie fait écho celui d'autogestion au Brésil. Ainsi Singer fait-il du concept d'autogestion un élément central de sa théorie. De même, pour Faria, il s'agit d'un enjeu central, qui souligne la nécessaire autonomie de décision de chaque unité de travail. Mais, tandis que pour Singer l'ES serait l'embryon d'une économie socialiste autogérée à venir, le sociologue Faria conçoit l'ES comme s'inscrivant dans une autogestion partielle qui ne vise pas de changement complet de société. 
Ces débats sur les rapports à l'Etat sont liés à ceux qui portent sur les transformations d'ordre culturel induites par l'ESS. Le changement social peut reposer sur les interventions possibles dans l'espace public des entités qui appartiennent à l'ESS, engendrant l'introduction de nouveaux thèmes dans le débat public. Ainsi, Laville porte son attention sur les interventions des associations dans l'espace public et sur leur capacité d'interpellation des pouvoirs publics. La prise en compte de l'apport de l'économie solidaire à la construction d'une société démocratique est également soulignée par Singer, qui envisage l'économie solidaire comme pouvant conduire à "l'extension de la démocratie » à partir de la participation politique des citoyens dans l'espace public. Ces conceptualisations pointent les apports de l'ESS dans le processus général d'approfondissement de la démocratie, par le renouvellement des thèmes débattus dans l'espace public qu'elle induit et par la participation d'un nombre croissant de citoyens à ces débats. Mais Singer insiste aussi sur les changements d'ordre culturel engendrés au niveau individuel par la participation à une organisation autogérée. Ainsi, pour Singer, l'autogestion s'inscrit dans un processus éducatif. De même, pour Draperi, c'est le changement à l'échelle individuelle qui est privilégié; le principe de double qualité doit être au service d'une finalité éducative, ce qui fait que l'économie sociale est simultanément "un mouvement économique qui s'appuie sur l'éducation et un mouvement éducatif qui s'appuie sur l'économie» (Draperi, 2007, p. 256). Il rejoint en cela Faria, qui place le dépassement de la distinction entre décideurs et exécutants comme l'un des critères essentiels des organisations sociales productives. Néanmoins, ces vertus éducatives ne constitueraient qu'un bénéfice secondaire pour Prades, qui place la capacité d'autodétermination des acteurs de l'ESS comme élément essentiel du changement social. Cependant, au-delà des différences énoncées plus haut, des points de convergence apparaissent nettement. Les théoriciens de l'ESS refusent de dissocier la dimension économique des rapports sociaux qui les sous-tendent, et ce faisant ils contribuent pleinement à l'élaboration d'un nouveau modèle culturel. Ce concept, initialement introduit par Alain Touraine dans les années 70, a été repris par Guy Bajoit pour désigner «l'ensemble des principes ultimes de sens qu'une collectivité humaine invoque et inculque à ses membres, à une époque donnée, pour donner du sens à leur vie en société, pour leur donner le sentiment que les solutions apportées aux grands problèmes vitaux de la vie collective sont de "bonnes solutions" et qu'en se soumettant à ces contraintes sociales, ils auront une "vie bonne", qui répondra à leurs besoins les plus profonds »(Bajoit, 2003, p. 56). Les quatre problèmes de la vie collective que repère Guy Bajoit sont: la production de richesse, l'ordre politique interne, le contrat social et le problème de la socialisation ${ }^{(2)}$. Plusieurs modèles culturels peuvent coexister dans une société, mais l'un d'eux est toujours prédominant. Depuis les années 70, de nouvelles solutions ont été apportées à ces quatre problèmes vitaux qui traduisent l'émergence d'un nouveau modèle culturel, de type identitaire, dénommé « modèle culturel de la modernité subjectiviste ». Nous présentons dans le tableau 1 les caractéristiques de ce nouveau modèle culturel en plaçant en parallèle le modèle culturel sousjacent aux théories de l'ESS.

(2) Notons que Guy Bajoit ajoute un cinquième problème, lié à la diplomatie, écarté de notre analyse car il ne présente que peu de pertinence pour notre sujet. 


\section{Tableau 1}

\begin{tabular}{|l|l|l|}
\hline Problèmes vitaux & $\begin{array}{l}\text { Modèle culturel } \\
\text { de la modernité subjectiviste }\end{array}$ & $\begin{array}{l}\text { Modèle culturel } \\
\text { de la modernité solidaire }\end{array}$ \\
\hline Système de production & $\begin{array}{l}\text { Modèle de production capitaliste } \\
\text { néolibéral mondialisé avec } \\
\text { une distinction nette } \\
\text { entre décideurs et exécutants }\end{array}$ & $\begin{array}{l}\text { Modèle de production reposant } \\
\text { sur l'autogestion ou la double } \\
\text { qualité avec une visée éducative }\end{array}$ \\
\hline Ordre politique & $\begin{array}{l}\text { Démocratie d'experts } \\
\text { avec des citoyens passifs }\end{array}$ & $\begin{array}{l}\text { Démocratie participative } \\
\text { avec une recherche d'émancipation } \\
\text { des individus }\end{array}$ \\
\hline Contrat social & $\begin{array}{l}\text { Etat social actif } \\
\text { Etat social fort avec un soutien } \\
\text { étatique possible aux entités } \\
\text { de l'ESs }\end{array}$ \\
\hline Type de socialisation & $\begin{array}{l}\text { Primauté du droit des individus } \\
\text { avec la recherche d'une } \\
\text { autoréalisation identitaire }\end{array}$ & $\begin{array}{l}\text { Primauté de l'entraide associée } \\
\text { à une capacité d'autodétermination }\end{array}$ \\
\hline
\end{tabular}

Les points de convergence des recherches portant sur l'ESS dans les deux pays peuvent ainsi être synthétisés à travers leur contribution commune à la mise en évidence d'entités participant à l'élaboration d'un nouveau modèle culturel au sens où l'entend Touraine. Le principe de socialisation est alors non plus centré sur la défense des droits individuels et le besoin de réalisation personnelle des individus, mais sur leur besoin d'entraide et leur capacité d'autodétermination, qui apparaissent à travers leur participation à la gestion d'activités économiques à une échelle locale. Le modèle de production de ces entités s'appuie sur le socle de la double qualité ou de l'autogestion au Brésil, qui s’inscrit dans une visée d'émancipation des individus. La proximité des projets éthico-politiques sous-tendant les conceptualisations des différents auteurs présentés ici se retrouve dans la construction d'un champ de recherche qui prend comme objet principal les entités participant aux quatre problèmes vitaux du modèle culturel de la modernité solidaire défini ci-dessus. Cet objet permet de poser les bases d'un champ de recherche vers lequel convergent diverses disciplines qui, par leurs apports conjoints, peuvent contribuer à terme à la construction d'une nouvelle discipline.

\section{Conclusion}

Nous avons montré que différentes conceptions du changement social soustendent les approches de l'ESS au Brésil et en France, mais des similarités ont aussi été constatées, et les points de rapprochement soulignent que des débats similaires peuvent se forger dans des contextes très différents. En cernant au plus près les enjeux de ces débats théoriques, nous avons souhaité contribuer à une meilleure compréhension de la dynamique interne des travaux académiques dans le domaine de l'ESS en soulevant des caractères qui nous paraissaient essentiels pour comprendre le lien entre les conceptualisations de l'ESS et le changement social. Nous avons choisi de comparer les recherches issues de deux pays où les contributions théoriques émanant du milieu académique ont atteint un degré de développement significatif. 
A travers le choix des auteurs mentionnés au fil de cette contribution se dessine une vue nécessairement parcellaire de la recherche en ESS, et de nombreux autres auteurs auraient mérité d'être cités. Néanmoins, cette première contribution permet de confronter les pensées des auteurs de ces deux pays et de cerner quelques enjeux essentiels. Du côté brésilien, les auteurs mettent en avant l'autogestion comme principe entrant en contradiction avec le reste de la société. Pour autant, le rôle de l'Etat apparaît important pour le développement de ce secteur. Du côté français, c'est l'autonomie qui est valorisée, mais on insiste également sur le rôle de l'Etat comme possible appui. La synthèse a permis de rapprocher les conceptualisations propres à chaque pays en soulignant leur contribution à la mise en évidence d'entités participant à l'élaboration d'un nouveau modèle culturel au sens où l'entend Touraine, dans lequel le principe de socialisation essentiel repose sur le besoin d'entraide et la capacité d'autodétermination de l'individu. 


\section{BibLIOgRAPHIE}

Arruda M., 2000, « Um novo humanismo para uma nova economia », in Kraychete, G., Lara F., Costa B., Economia dos setores populares: entre a realidade e a utopia, Petrópolis, Editora Vozes. Arruda M., 2003, «Socioeconomia solidária », in Cattani A. D., A outra economia, Porto Alegre, Veraz Editores.

Arruda M., 2005, « Redes, educação e economia solidária: novas formas de pensar a educação de jovens e adultos", in Kruppa S. P. (éd), Economia solidária e educação de jovens e adultos, Brasília, ministério da Educação.

Bajoit G., 2003, Le changement social: approche sociologique des sociétés occidentales contemporaines, Paris, Armand Colin.

Carvalho de França Filho G., 2003, « L'économie solidaire en France et au Brésil: regards croisés », Revue du Mauss, vol. 1, nº 21.

Draperi J.-F., 2007, Comprendre l'économie sociale: fondements et enjeux, Paris, Dunod.

Draperi J.-F., 2005, L'économie sociale: utopies, pratiques, principes, Presses de l'économie sociale, Montreuil.

Eme B., Laville J.-L., 1988, Les petits boulots en question, Syros, Paris.

Faria (de) J. H., 2009, Gestão participativa: relações de poder e de trabalho nas organizações, São Paulo, Editora Atlas S.A.

Gaiger L. I., 2000, «Sentidos e possibilidades da economia solidária hoje », in Kraychete G., Lara F., Costa B., Economia dos setores populares: entre a realidade e a utopia, Petrópolis, Editora Vozes.

Gaiger L. I. (dir), 2004, Sentidos e experiências da economia solidária no Brasil, Porto Alegre, Editora da UFRGS.

Hély M., 2009, Les métamorphoses du monde associatif, Presses universitaires de France.
Hiez D., Lavillunière E. (dir.), 2013, Vers une théorie de l'économie sociale et solidaire, De Boeck, p. 7-16.

Laville J.-L., 2005, Action publique et économie solidaire: une perspective internationale, Editions Erès.

Laville J.-L., 2001, « Vers une économie sociale et solidaire? », Recma, $\mathrm{n}^{\circ} 281$.

Laville J.-L., 1994, L'économie solidaire: une perspective internationale, Desclée de Brouwer.

Leite M. de P., Araújo A. C., Lima J., 2011, Relatório final: A crise do trabalho e as experiências de geração de emprego e renda: as distintas faces do trabalho associado e a questão de gênero, Campinas. Consultable en ligne: www.ifch.unicamp.br/gptrabalho/node/4.

Leite M. de P., 2011, « Cooperativas e trabalho: um olhar sobre o setor de reciclagem e fábricas recuperadas em São Paulo ", in Anais do II Seminário internacional organização e condições do trabalho moderno, Campinas. Consultable en ligne: http://portal.anpocs.org/portal/index. php?option=com_docman\&task=doc_view\&gid $=1275 \&$ Itemid $=353$.

Prades J., 2013, L’utopie réaliste: le renouveau de l'expérience coopérative, L'Harmattan.

Prades J., 2006, Compter sur ses propres forces: initiatives solidaires et entreprises sociales, Editions de l'Aube.

Singer P., 2000a, Uma utopia militante: repensando o socialismo, Petrópolis, Vozes, $2^{\mathrm{e}}$ éd.

Singer P., 2000b, Economia socialista, São Paulo, Editora Fundação Perseu Abramo.

Singer P., 2003, "Economia solidária ", in Cattani A. D., A outra economia, Porto Alegre, Veraz Editores. 